\title{
Cortical, subcortical and brain stem connections of the cerebellum via the superior and middle cerebellar peduncle in the rat
}

\author{
Safiye Çavdar ${ }^{\mathrm{a}, *}$, Merve Özgür ${ }^{\mathrm{a}}$, Yasemin Kuvvet ${ }^{\mathrm{a}}$, Hüsniye Bay ${ }^{\mathrm{b}}$ and Evren Aydogmus ${ }^{\mathrm{c}}$ \\ ${ }^{a}$ Department of Anatomy, School of Medicine, Koç University, Istanbul, Turkey \\ ${ }^{\mathrm{b}}$ Department of Anatomy, School of Medicine, Marmara University, Istanbul, Turkey \\ ${ }^{\mathrm{c}}$ Department of Neurosurgery, Dr. Lütfi Kirdar Kartal Education and Research Hospital, Istanbul, \\ Turkey
}

Received 29 March 2018

Accepted 14 May 2018

\begin{abstract}
The role of cerebellum in coordination of somatic motor activity has been studied in detailed in various species. However, experimental and clinical studies have shown the involvement of the cerebellum with various visceral and cognitive functions via its vast connections with the central nervous system. The present study aims to define the cortical and subcortical and brain stem connections of the cerebellum via the superior (SCP) and middle (MCP) cerebellar peduncle using biotinylated dextran amine (BDA) and Fluoro-Gold (FG) tracer in Wistar albino rats. 14 male albino rats received 20-50-nl pressure injections of either FG or BDA tracer into the SCP and MCP. Following 7-10 days of survival period, the animals were processed according to the related protocol for two tracers. Labelled cells and axons were documented using light and fluorescence microscope. The SCP connects cerebellum to the insular and infralimbic cortices whereas, MCP addition to the insular cortex, it also connects cerebellum to the rhinal, primary sensory, piriform and auditory cortices. Both SCP and MCP connected the cerebellum to the ventral, lateral, posterior and central, thalamic nuclei. Additionally, SCP also connects parafasicular thalamic nucleus to the cerebellum. The SCP connects cerebellum to basal ganglia (ventral pallidum and clastrum) and limbic structures (amygdaloidal nuclei and bed nucleus of stria terminalis), however, the MCP have no connections with basal ganglia or limbic structures. Both the SCP and MCP densely connects cerebellum to various brainstem structures. Attaining the knowledge of the connections of the SCP and MCP is important for the diagnosis of lesions in the MCP and SCP and would deepen current understanding of the neuronal circuit of various diseases or lesions involving the SCP and MCP.
\end{abstract}

Keywords: Superior and middle cerebellar peduncle, cortical, subcortical, brainstem connections

\section{Introduction}

Cerebellum communicates with the other parts of central nervous system via the superior (SCP), middle (MCP), and inferior (ICP) cerebellar peduncles. The SCP (brachium conjunctivum) contain primarily

\footnotetext{
${ }^{*}$ Corresponding author: Safiye Çavdar, Department of Anatomy, School of Medicine, Koç University, 34450 Sarıyer, Istanbul, Turkey. Fax: 9021633810 73; E-mail: scavdar@ku.edu.tr.
} 
efferent pathways that emerge from the deep cerebellar nuclei (i.e., dentate, emboliform, fastigial and globose), pass through the ipsilateral SCP into the dorsal pons, decussate at the level of the inferior colliculus, travel to the thalamus and then to the contralateral cerebral cortex [19]. The majority of the fibers of the MCP are afferent fibers from the contralateral pontine nuclei [14]. Thus, conveys cortical fibers via the pons to the cerebellum (cortico-ponto-cerebellar tract).

Cerebellum was considered as an organ related to motor functions, however studies have shown its relations with cardiovascular, respiratory, gastrointestinal functions, further, cognition, working memory, emotion, reading skills and even with immune system [2,8,10,28,29,33,35,36]. Further, studies have shown the involvement of cerebellum in cognitive processes, such as processing and coordinating information [22].

The role of cerebellum in many neurological diseases, such as Friedreich's ataxia [1,3,18], Parkinson's disease [15], schizophrenia [17,32], progressive supranuclear palsy $[9,15,16,21,30]$ and Joubert syndrome $[6,20,23,25,27]$ has been demonstrated using both experimental animal models and human imaging.

Growing understanding of how the cerebellum communicate with other areas of the central nervous system has enriched our understanding of the cerebellar circuits, and the areas that cerebellar activity can influence. In the present study we aimed to define the cortical and subcortical and brain stem connections of the cerebellum via the SCP and MCP using biotinylated dextran amine (BDA) and Fluoro-Gold (FG) tracer in Wistar albino rats.

\section{Materials and methods}

Wistar albino rats weighing $250-400 \mathrm{~g}$ were fed with a standard laboratory rat chow and tap water ad libitum, and housed in Plexiglass cages with a 12-h light/dark cycle in a temperature-controlled room $\left(20 \pm 30^{\circ} \mathrm{C}\right)$. The Institutional Animal Care and Use Committee of Koç University approved all procedures. A total of 14 animals were used for the BDA and FG injections to the SCP and MCP. Of the 14 injections $n=7$ were to the SCP and $n=7$ were made to the MCP. Rats were anaesthetized deeply with ketamine $(100 \mathrm{mg} / \mathrm{kg}$, intraperitoneally) and chlorpromazine $(1 \mathrm{mg} / \mathrm{kg}$, intraperitoneally). The heads of the animals were placed in a stereotaxic frame (Stoelting Model 51600, Wood Dale, IL,

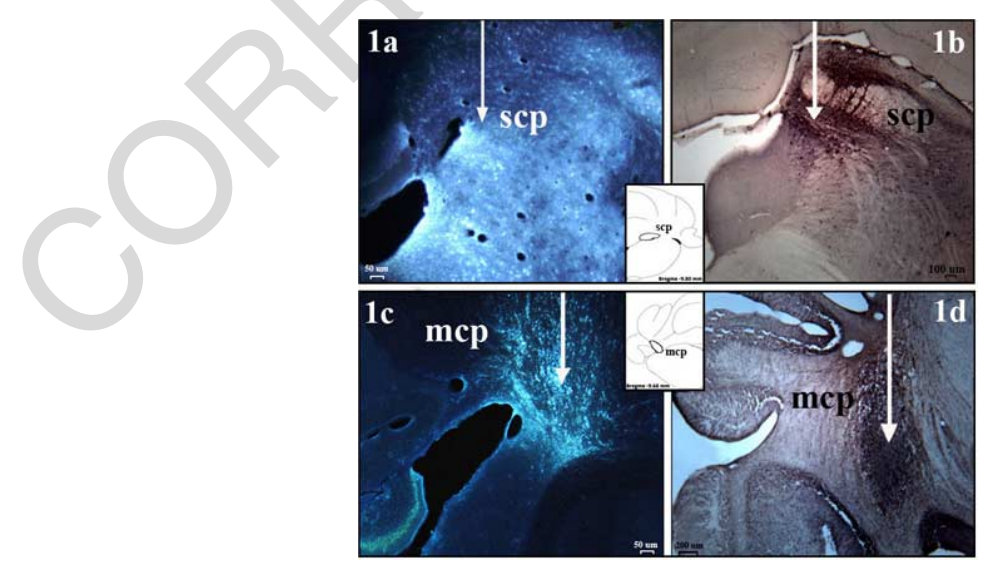

Fig. 1. (a) FG injection site to the SCP, (b) BDA injection site to the SCP, (c) FG injection site to the MCP, and (d) BDA injection site to the MCP. A drawing of the coronal section of the cerebellum was added on the center of the figure for orientation. 
USA). The scalp was incised longitudinally, and the skull was exposed between lambda and bregma. A small hole was drilled in the skull at a position appropriate for the unilateral injection of tracer into the either the SCP or MCP. The injections to the SCP were made at a level $-9.84 \mathrm{~mm}$ posterior to bregma, $6.8 \mathrm{~mm}$ ventral to the surface of the skull and $1.3 \mathrm{~mm}$ lateral to the midline (Fig. 1a, b). The injections to the MCP were made at a level $-9.96 \mathrm{~mm}$ posterior to bregma, $6.6 \mathrm{~mm}$ ventral to the surface of the skull and $4.3 \mathrm{~mm}$ lateral to the miline (Fig. 1c, d).

\subsection{FG injections}

The $0.1 \mu 1$ of FG solution (2\%, FluoroChrome Inc., Englewood, CO, USA) with a rate of $0.02 \mu 1 / \mathrm{min}$ was pressure injected into SCP and MCP. After 5-7 days' surviving animals were deeply anaesthetized with ketamine $(100 \mathrm{mg} / \mathrm{kg}$, intraperitoneally) and perfused transcardially with $250-300 \mathrm{ml}$ of heparinized saline solution, followed by $2.5 \%$ paraformaldehyde in $0.1 \mathrm{M}$ phosphate buffer $(400-450 \mathrm{ml})$. Brains were removed and post fixed in the same solution for $24 \mathrm{~h}$ at $4^{\circ} \mathrm{C}$. Coronal sections $(40 \mu \mathrm{m})$ were cut on a vibrotome (Leica). Every fourth section was placed on gelatin subbed glass slides, dehydrated and cleared, covered with DPX and examined under a fluorescence microscope.

\subsection{BDA injection}

The $0.2 \mu \mathrm{l}$ of BDA solution (10\% BDA in 0.1 M PBS, Molecular Probes, Eugene, OR, USA) with a rate of $0.02 \mu \mathrm{l} / \mathrm{min}$ was pressure injected into STN. After 10 days survival the animals underwent the same procedure to FG method. Coronal serial sections $(40 \mu \mathrm{m})$ were cut on a vibrotome (Leica) and free floating sections were collected into a well plates. Immunohistochemistry was applied to every fourth section. The BDA was visualized in sections using a standard avidin-biotin complex (Vectastain Elite, ABC kit) horseradish peroxidase staining procedure. Sections were transferred to PBS and washed for $10 \mathrm{~min}$, three times, and then incubated 3 hours at room temperature in Vectastain working solution which was prepared 30 min before use and contained Reagant A (Avidin DH, 1 drop) and Reagent B (Biotinylated Horseradish Peroxidase H, 1 drop) in $5 \mathrm{ml}$ PBS. After three additional $10 \mathrm{~min}$ PBS washes, tissue was then exposed to diaminobenzidine (DAB, Vector Labs). The reaction was stopped with distilled water, and washed for $5 \mathrm{~min}$. After the dehydration process, the sections were mounted with Entallan.

The FG and BDA results presented are selected from animals in which the centers of the injection sites were optimal with minimal contamination of the adjacent structures or along the pipette tract.

\section{Results}

In the present study FG and the BDA tracer was injected to the SCP and MCP. Subsequent to the FG and BDA injections, consistent labeled cells and axons in various cortical, subcortical and brain stem structures were documented. The density of connections are shown in Table 1.

\subsection{The BDA and FG injection to the SCP}

Cortical connections of the cerebellum via the SCP were with the infralimbic (IL) and insular (ICx) cortices (Fig. 2). The insular and infralimbic cortex showed both FG and BDA labelled cells (Table 1). 
Table 1

Cortical, subcortical and brain stem connections of cerebellum via SCP and MCP

\begin{tabular}{|c|c|c|}
\hline Cortical, subcortical and brainstem & $\begin{array}{l}\text { Connections of fibers } \\
\text { of the SCP }\end{array}$ & $\begin{array}{l}\text { Connections of fibers } \\
\text { of the MCP }\end{array}$ \\
\hline \multicolumn{3}{|l|}{ Cortical } \\
\hline Insular (ICx) & $*$ & $* *$ \\
\hline Infralimbic (IL) & $*$ & - \\
\hline Rhinal (Ect, PRh) & - & $*$ \\
\hline Primary sensory $(\mathrm{PrS})$ & - & $*$ \\
\hline Piriform (Pir 1, 2, 3) & - & $* *$ \\
\hline Auditory (AuD) & - & $*$ \\
\hline \multicolumn{3}{|l|}{ Subcortical } \\
\hline \multicolumn{3}{|l|}{ Thalamic } \\
\hline Ventral (VL,VA, VM) & * & $* *$ \\
\hline Parafaicular (PF, RPF) & $*$ & - \\
\hline Central (CM, PC) & * & $*$ \\
\hline Posterior (Po) & $*$ & $* *$ \\
\hline Lateral (LD, LP) & $*$ & $*$ \\
\hline \multicolumn{3}{|l|}{ Basal ganglia } \\
\hline Ventral pallidum (VP) & $*$ & - \\
\hline Clastrum $(\mathrm{DCl}, \mathrm{VCl})$ & $*$ & - \\
\hline \multicolumn{3}{|l|}{ Amygdala } \\
\hline Central (CeL, CeM, CeC) & & - \\
\hline Lateral (LaVL) & & - \\
\hline Basolateral (BLA) & & - \\
\hline Bed nucleus of stria terminalis (BST) & & * \\
\hline \multicolumn{3}{|l|}{ Subthalamus } \\
\hline Subthalamic nucleus (STh, PSTh) & * & - \\
\hline Zona incerta (ZI) & $*$ & $*$ \\
\hline
\end{tabular}

The insular and infralimbic cortices connections were bilateral, moderate on the ipsilateral and sparse on the contralateral side. No other cortical regions had direct connections with the cerebellum via SCP.

The SCP connects cerebellum to subcortical structures; the thalamus, hypothalamus, subthalamus, limbic and basal ganglia (Table 1). The cerebellum was connected to ventral (VL, VA and VM), lateral (LD, LP), posterior (Po), central (CM, PC), and parafasicular (PF) thalamic nuclei via the SCP (Table 1) (Fig. 3). All thalamic connections were bilateral, moderate on the ipsilateral and sparse on the contralateral side. The details of the hypothalamo-cerebellar connections is under review for publication.

The FG injections to the SCP showed connections of the cerebellum with ventral pallidum (VP) and clastrum (Cl) (Fig. 2). Labelled cells were strictly observed on the ipsilateral side. The BDA and FG injections into the SCP showed connections of the cerebellum with the central $(\mathrm{CeL}, \mathrm{CeM}, \mathrm{CeC})$ lateral (LaVL) and basolateral (BLA) amygdaloidal nuclei and the bed nucleus of stria terminalis (BST) (Fig. 4a, b). Labelled cells were observed on the contralateral side of the injection. Further, weak FG and BDA labelled cells were also observed on the ipsilateral subthalamic nucleus (STN) and zona incerta (ZI) (Table 1).

Dense connection between the cerebellum and the brainstem were observed. The FG and BDA labelled cells were present at the Darkawich (Dk), Edinger Westphal (EW), Intersitial nucleus of Cajal (INC), 
Table 1

(Continued)

\begin{tabular}{|c|c|c|}
\hline Cortical, subcortical and brainstem & $\begin{array}{l}\text { Connections of fibers } \\
\text { of the SCP }\end{array}$ & $\begin{array}{l}\text { Connections of fibers } \\
\text { of the MCP }\end{array}$ \\
\hline \multicolumn{3}{|l|}{ Brainstem } \\
\hline Red nucleus (RMC, RPC) & $* *$ & $* * *$ \\
\hline prerubral (PR), \& Pararubral (PaR) & $*$ & * \\
\hline Dorsal raphe (DR, MnR, CLi) & $*$ & $*$ \\
\hline Parabrachial (PBP, MPB, LPB) & $*$ & $*$ \\
\hline Darkawich (Dk) & $*$ & - \\
\hline Edinger-Westphal (EW) & $*$ & - \\
\hline Anterior pretectal (APT) & $* *$ & * \\
\hline Substantia nigra $(\mathrm{SN})$ & - & $*$ \\
\hline Reticular nucleus (IRt, PCRt, Gi, mRt) & * & * \\
\hline Interstitial nucleus of Cajal (InC) & $*$ & - \\
\hline Periaquaductal gray (PAG, LPAG) & $*$ & * \\
\hline Trigeminal spinal (Sp5, Pr5) & - & $*$ \\
\hline Tegmental (MiTg, RtTg, PTg) & $*$ & $*$ \\
\hline Vestibular (SpVe, MVe) & $*$ & $*$ \\
\hline Geniculate (MG, VG) & - & $* *$ \\
\hline Inferior olivary (IOD, IOC, IOB) & $*$ & $* *$ \\
\hline Pontine (Pn, POn) & - & $* * *$ \\
\hline Inferior colliculus (ECIC) & . & $*$ \\
\hline Locus cerleus (LC) & & - \\
\hline Dorsal cohlear (DCD) & & $*$ \\
\hline Superior colliculus (SuC) & & $*$ \\
\hline Cuneiform (CnF) & & $*$ \\
\hline Oculomotor (3PC) & - & $*$ \\
\hline \multicolumn{3}{|l|}{ Cerebellar } \\
\hline Lateral (Lat, LatPC) & $*$ & $* * *$ \\
\hline Interpositus (IntA, IntP) & $*$ & $* *$ \\
\hline Medial (Med) & $*$ & $*$ \\
\hline Cerebellar cortex $(\mathrm{CB})$ & $*$ & $*$ \\
\hline
\end{tabular}

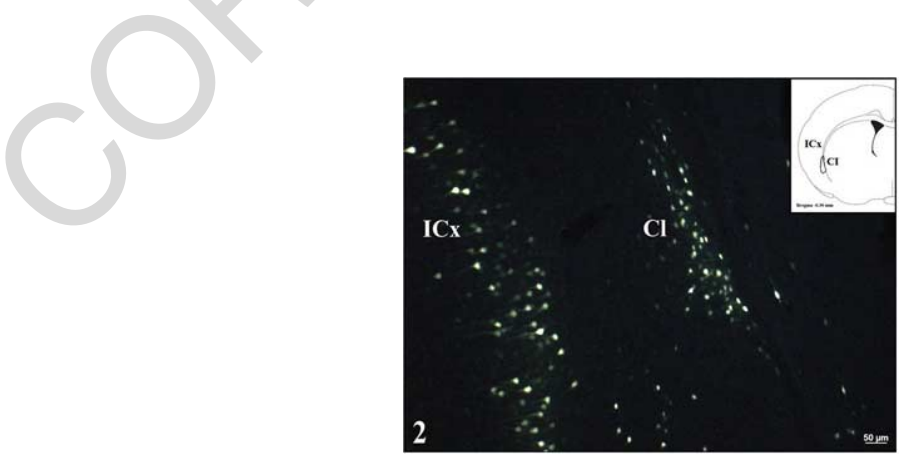

Fig. 2. Labelled cells in the V layer of insular cortex and the claustrum subsequent to FG injection into the SCP. A drawing of the coronal section of the brain was added on the right corner of the figure for orientation. (ICx insular cortex, $\mathrm{Cl}$ clastrum.) 

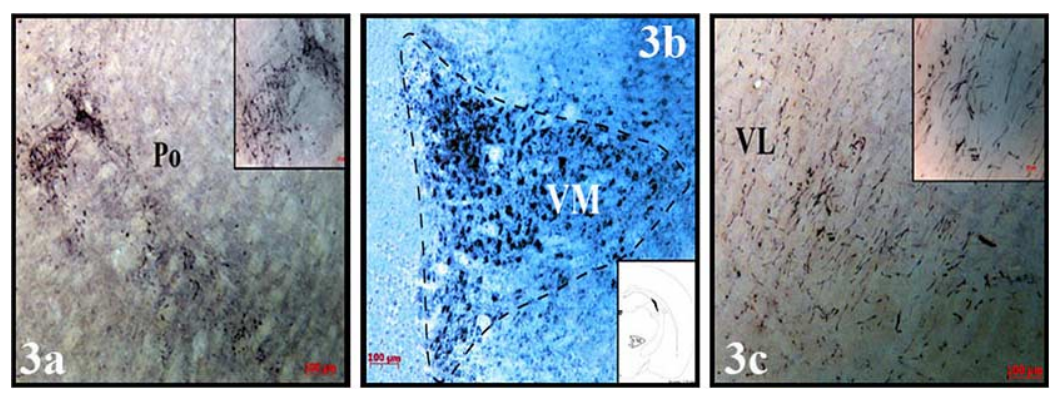

Fig. 3. Examples of BDA and FG labelled cells and axons in the thalamic nuclei subsequent to injection into the SCP and MCP. (a) Labelled axons in the posterior thalamic nuclei (Po) and magnified image subsequent to BDA injections into the MCP, (b) labelled cells in the ventromedial thalamic nuclei (VM) subsequent to BDA injections into the SCP, (c) labelled axons in the ventral lateral $(\mathrm{VL})$ thalamic nucleus subsequent to BDA injections into the MCP.

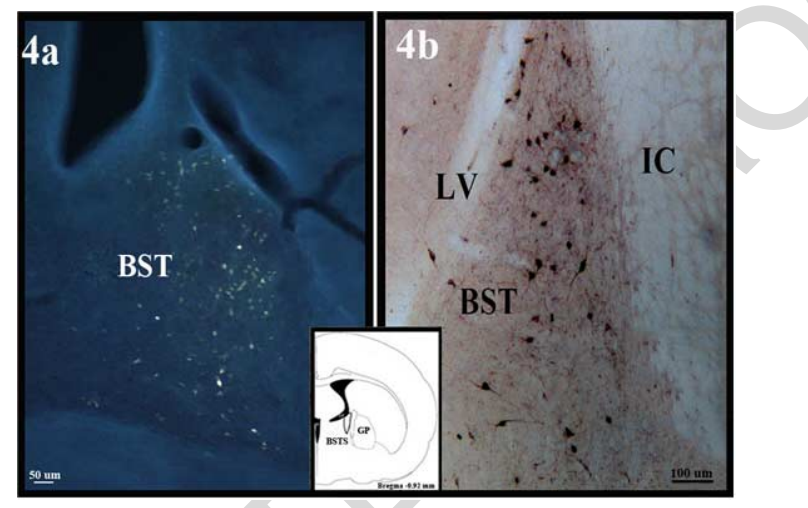

Fig. 4. (a) FG and (b) BDA labelled cells in the bed nucleus of stria terminalis (BST) subsequent to SCP. A drawing of the coronal section of the brain was added on the center of the figure for orientation.

reticular (IRt, LPGi), periaqueductal gray (PAG), tegmental (RtTg), red (RMC), rubral (PR, PaR), raphe (DR, MnD, PMnR, CLi), parabrachial (PB), anterior pretectal (APT), vestibular nuclei (SpVe), and substantia nigra $(\mathrm{SN})$. The density of the connections between the cerebellum and the brain stem nuclei that passes within the SCP are shown in Table 1.

\subsection{The BDA and FG injection to the $M C P$}

Cortical connections of the cerebellum via the MCP were with the insular (GI, DI), rhinal (Ect, PRh) primary sensory (PrS), piriform (Pir 1, 2, 3) and auditory cortices (AuCx). All cortical afferents to the cerebellum were bilateral, moderate on the ipsilateral and sparse on the contralateral side.

The cerebellum was connected to ventral (VL, VA), Po, LP and thalamic reticular nuclei via the MCP (Fig. 3a, b) (Table 1). All thalamic connections were ipsilateral to the injection side. The BDA and the FG injections into the MCP also showed connections between the cerebellum and the hypothalamic nuclei. The hypothalamic-cerebellar connections is under review for publication. No labelled FG or BDA cells or axons were observed in the basal ganglia and amygdaloid nuclei. However, dense connections between the cerebellum and the brainstem via the MCP were observed. These included trigeminal spinal (Sp5, Pr5), periaquaductal gray (LPAG), tegmental (MiTg, RtTg, PTg), pontine (Pn, POn), reticular nucleus (PCRt, Gi), vestibular (SpVe, MVe), parabrachial (LPB, MPB), inferior olivary (IOD, IOC, IOB), 

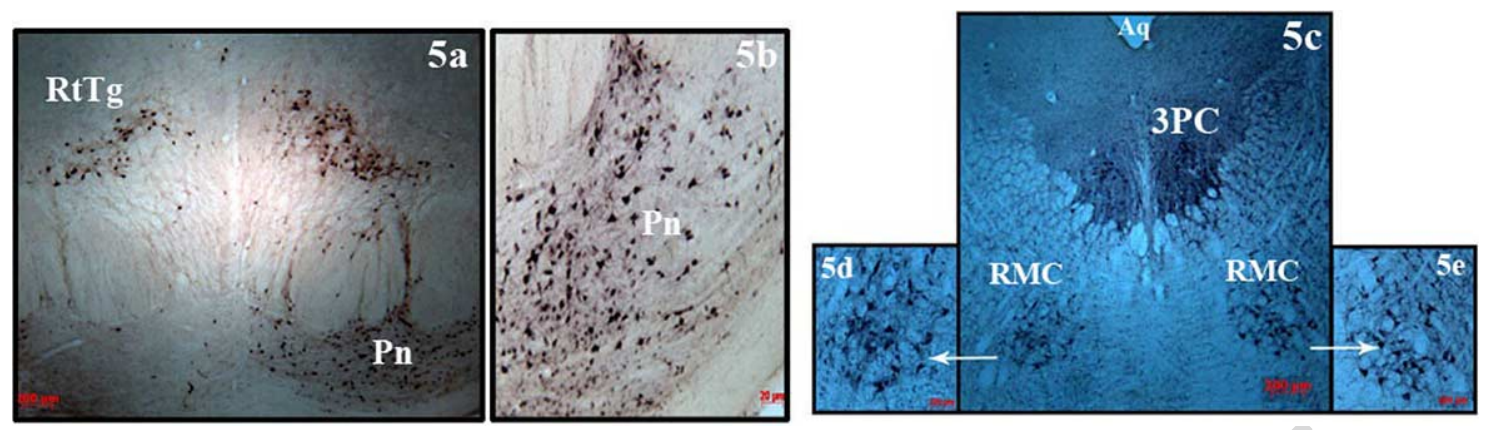

Fig. 5. Examples of FG and BDA labelled cells in the various brain stem nuclei subsequent MCP injections. (a) BDA labelled cells in the pontine (Pn) and reticulotegmental (RtTg) nucleus. (b) Magnified BDA labelled neurons in the pontine nucleus. (c) BDA labelled cells in the red nucleus (RMC) and oculomotor nucleus (3PC) subsequent to MCP injections. (d), (e) Magnified image of the BDA labelled RMC neurons.

geniculate (MG, VG), pontine (Pn, POn), dorsal raphe (DR), inferior colliculus (ECIC), red nucleus (RMC, RPC) (Fig. 5a, b, c, d, e), anterior pretectal (APT), dorsal cochlear (DCD), superior colliculus (SC), cuneiform $(\mathrm{CnF})$ and prerubral $(\mathrm{PR})$ nuclei.

Subsequent to FG and BDA injections into the SCP and the MCP labelled cells were observed in the lateral (Lat), medial (Med), anterior interposed (IntA), and posterior interposed (IntP) nuclei and in all layers of the cerebellar cortex ( $\mathrm{Cb} 1-9)$.

\section{Discussion}

\subsection{Major findings}

Both the SCP and the MCP are directly connected to the insular cortex. Addition to the insular cortex MCP is connected to the rhinal, sensory, piriform and auditory cortices. Both SCP and MCP are connected to the ventral, lateral, central and posterior thalamic nuclei. The SCP connects cerebellum to ventral pallidum, clastrum and amygdaloidal nuclei, however, the MCP have no connections with basal ganglia or limbic structures. Both the SCP and MCP densely connects cerebellum to various brainstem structures (see Table 1). These connections on various novel routes can change the conceptual architecture of the cerebellar circuitry. Further, can be useful in defining the neuronal circuit of various diseases involving the SCP and MCP.

\subsection{Cortical connections of the cerebellum}

It has been shown that cortico-cerebellar system consists of layer V neurons of the cerebral cortex connect with the cerebellar cortex via relays in the pontine nuclei [4]. The MCP connects the contralateral pontine nuclei to the cerebellum [14]. Thus, conveys cortical fibers via the pons to the cerebellum (cortico-ponto-cerebellar tract). The results of the present study showed both MCP and SCP connected cerebellum specific regions of the cortex. Studies have shown that cerebellar cortex return projections to the cerebral cortex via relays in the cerebellar nuclei and the thalamus [12]. Sasaki et al. [26] demonstrated short-latency evoked potentials in the anterior parietal cortex following electrical stimulation of the cerebellum in the cat. Further, anatomical studies performed in monkeys have revealed that left inferior prefrontal regions and left premotor areas of the frontal lobes are reciprocally connected with the 
right hemisphere of the cerebellum conveyed by the SCP and the MCP, respectively. The present study addition to the indirect connections of the cerebellum with the cortex via relay nuclei, also showed direct cerebello-cortical connections. The infralimbic and insular cortices was connected via the SCP and rhinal, insular, primary sensory piriform and auditory cortices was connected via MCP. The connections from the cerebral cortex to the pontine nuclei are the main routes through which the cerebral cortex supplies the cerebellum with information. The direct connections can be an alternative route through which cortical information arrives at the cerebellum.

\subsection{Subcortical connections of the cerebellum}

Miller and Strominger [13] made a unilateral electrolytic lesion in the right SCP or to the dentate nucleus of monkey and observe lesion in the VPL, VL, VA, Cl and PCn, nucleus ruber, reticular formation, nucleus reticularis tegmentalis and nucleus raphe pontis and magnus and nucleus gigantocellularis. The present study confirms the connection of the cerebellum with VL, VA and PC thalamic connections, however we have not detected its connections with the VPL thalamic nuclei. Addition to the above thalamic nuclei SCP and MCP also connects the VM, PF, LD and Po thalamic nuclei. Striking result of the present study is that the majority of cerebellar connections were with motor higher-order thalamic nuclei (VA, Po, CM, VM, PF and LP), and only one with motor first-order thalamic nuclei (VL). Thus, cerebellum can be in a position to influence large areas of the neocortex by modulating some of the motor higher-order thalamic nuclei.

We confirm the brainstem results (nucleus ruber, reticular formation, nucleus reticularis tegmentalis and nucleus raphe pontis and magnus and nucleus gigantocellularis) of Miller and Strominger [13]. Additionally we also report connection of the cerebellum via the SCP and MCP with various brain stem structures which has not been reported earlier, these include; parabrachial, Darkawich, EdingerWestphal, anterior pretectal, interstitial nucleus of Cajal, substantia nigra, tegmental, trigeminal, vestibular, geniculate, inferior olivary, inferior colliculus, cuneiform, dorsal cochlear and superior colliculus (see Table 1).

Early studies using stimulation or ablation of the cerebellar structures have shown wide range of visceral responses (piloerection, changes in blood pressure, heart rate and respiration, alterations in smooth muscle tones [5,7,34]. Brainstem-cerebellar connections via the SCP and MCP can be responsible for the visceral responses. The influence of the majority of the brainstem-cerebellar connections is currently unknown extensive studies are necessary in this area.

Lesion of the SCP and MCP can be caused by various pathological conditions; infraction, degeneration, demyelination disease, toxic metabolic disease, trauma, tumors. In recent years due to the developments in the radiological techniques, numerous studies have shown the involvement of the SCP and $\mathrm{MCP}$ in various clinical conditions. Strong correlation between atrophy of the SCP and MCP with many disease were documented $[1,9,11,15,17,23,24,31]$. Thus, understanding the connections of the SCP and MCP is important for the diagnosis of lesions in the MCP and SCP. Cerebellum takes part in the higher order brain functions via its extensive connections. Being aware of the connection can be useful in defining the neuronal circuit of various diseases involving the SCP and MCP.

\subsection{Methodological remarks}

Although we aimed at small injections to SCP and MCP we still had contamination of the tracer to the neighboring regions. We used the same coordinate for all animals, also selected animal with similar 
weight and injected same amount of trace into the SCP or MCP we observed different density and number of labelled cells. Thus, we tried to overcome the former limitations by optimizing the study by increasing the number of experiment $(n=7)$.

\section{References}

[1] H. Akhlaghi, L. Corben, N. Georgiou-Karistianis, J. Branshaw, E. Storey, M.B. Delatycki et al., Superior cerebellar peduncle atrophy in Friedreich's ataxia correlates with disease symptoms, Cerebellum 10 (2011), 81-87. doi:10.1007/ s12311-010-0232-3.

[2] M.P. Alexander, S. Gillingham, T. Schweizer and D.T. Stuss, Cognitive impairments due to focal cerebellar injuries in adults, Cortex 48 (2012), 980-990. doi:10.1016/j.cortex.2011.03.012.

[3] I. Bombin, C. Arango and R.W. Buchanan, Significance and meaning of neurological signs in schizophrenia: Two decades later, Schizophr. Bull. 31 (2005), 962-977. doi:10.1093/schbul/sbi028.

[4] P. Brodal, Principles of organization of the monkey corticopontine projection, Brain Res. 148 (1978), 214-218. doi:10. 1016/0006-8993(78)90392-X.

[5] K. Chida, C. Iadecola, M.D. Underwood and D.I. Reis, A novel vasodepressor response elicited from the rat cerebellar fastigial nucleus: The fastigial depressor response, Brain Res. 370 (1986), 378-382. doi:10.1016/0006-8993(86)90498-1.

[6] R.J. Ferland, W. Eyaid, R.V. Collura, L.D. Tully, R.S. Hill, D. Al-Nouri et al., Abnormal cerebellar development and axonal decussation due to mutations in AHI1 in Joubert syndrome, Nat. Genet. 36 (2004), 1008-1013. doi:10.1038/ ng1419.

[7] D.E. Haines, E. Dietrichs, G.A. Mihailoff and E.F. Mcdonald, The cerebellar-hypothalamic axis: Basic circuits and clinical observations, Int. Rev. Neurobiol. 41 (1997), 83-107.

[8] M.J. Holmes, L.A. Cotter, H.E. Arendt, S.P. Cass and B.J. Yates, Effects of lesions of the caudal cerebellar vermis on cardiovascular regulation in awake cats, Brain Res. 31 (2002), 62-72. doi:10.1016/S0006-8993(02)02495-2.

[9] H. Kataoka, Y. Tonomura, T. Taoka and S. Ueno, Signal changes of superior cerebellar peduncle on fluid-attenuated inversion recovery in progressive supranuclear palsy, Parkinson Relat. Disord. 4 (2008), 63-65. doi:10.1016/j.parkreldis. 2007.03.001.

[10] U. Ladabaum, S. Minoshima, W.L. Hasler, D. Cros, W.D. Chey and C. Owyang, Gastric distention correlates with activation of multiple cortical and subcortical regions, Gastroenterology 120 (2001), 369-376. doi:10.1053/gast.2001.21201.

[11] J.L. Lewis, J.J. Lo Turco and P.R. Solomon, Lesions of the middle cerebellar peduncle disrupt acquisition and retention of the rabbit's classically conditioned nictitating membrane response, Behav. Neurosci. 101 (1987), 151-157. doi:10.1037/ 0735-7044.101.2.151.

[12] F.A. Middleton and P.L. Strick, Cerebellar output channels, Int. Rev. Neurobiol. 41 (1997), 61-82. doi:10.1016/S00747742(08)60347-5.

[13] R.A. Miller and N.L. Strominger, An experimental study of the efferent connections of the superior cerebellar peduncle in the rhesus monkey, Brain Res. 16 (1977), 237-250. doi:10.1016/0006-8993(77)90761-2.

[14] T. Naidich, H. Duvernoy, B. Delman, A. Sorensen, S. Kollias and E. Haacke, Duvernoy's Atlas of the Human Brain Stem and Cerebellum: High-Field MRI, Surface Anatomy, Internal Structure, Vascularization and 3 D Sectional Anatomy, Springer, Vienna, 2009.

[15] G. Nicoletti, C. Tonon, R. Lodi, F. Condino, D. Manners, E. Malucelli et al., Apparent diffusion of the superior cerebellar peduncle differentiates progressive supranuclear palsy from Parkinson's disease, Mov. Disord. 15 (2008), 2370-2376. doi: $10.1002 / \mathrm{mds} .22279$.

[16] M. Oka, S. Katayama, Y. Imon, T. Ohshita, Y. Mimori and S. Nakamura, Abnormal signals on proton density-weighted MRI of the superior cerebellar peduncle in progressive supranuclear palsy, Acta Neurol. Scand. 104 (2001), 1-5. doi:10. 1034/j.1600-0404.2001.00262.x.

[17] G. Okugawa, K. Nobuhara, T. Minami, K. Takase, T. Sugimoto, Y. Saito et al., Neural disorganization in the superior cerebellar peduncle and cognitive abnormality in patients with schizophrenia: A diffusion tensor imaging study, Prog. Neuropsychopharmacol. Biol. Psychiatry 30 (2006), 1408-1412. doi:10.1016/j.pnpbp.2006.05.014.

[18] E. Pagani, A. Ginestroni, R. Della Nave, F. Agosta, F. Salvi, G. De Michele et al., Assessment of brain white matter fiber bundle atrophy in patients with Friedreich ataxia, Radiology 25 (2010), 882-889. doi:10.1148/radiol.10091742.

[19] A. Parent, Carpenter's Human Neuroanatomy, 9th edn, Williams \& Wilkins, Baltimore, MD, 1996, pp. 612-613.

[20] M.A. Parisi, J.D. Pinter, I.A. Glass, K. Field, B.L. Maria, P.F. Chance et al., Cerebral and cerebellar motor activation abnormalities in a subject with Joubert syndrome: Functional magnetic resonance imaging (MRI) study, J. Child Neurol. 19 (2004), 214-218. 
[21] D.C. Paviour, S.L. Price, J.M. Stevens, A.J. Lees and N.C. Fox, Quantitative MRI measurement of superior cerebellar peduncle in progressive supranuclear palsy, Neurology 64 (2005), 675-679. doi:10.1212/01.WNL.0000151854.85743. C7.

[22] H. Picard, I. Amado, S. Mouchet-Mages, J. Olie and M. Krebs, The role of the cerebellum in schizophrenia: An update of clinical, cognitive, and functional evidences, Schizophr. Bull. 34 (2008), 155-172. doi:10.1093/schbul/sbm049.

[23] A. Poretti, E. Boltshauser, T. Loenneker, E.M. Valente, F. Brancati, K. Il'yasov et al., Diffusion tensor imaging in Joubert syndrome, AJNR Am. J. Neuroradiol. 28 (2007), 1929-1933. doi:10.3174/ajnr.A0703.

[24] P. Preziosa, M.A. Rocca, S. Mesaros, E. Pagani, J. Drulovic, T. Stosic-Opincal et al., Relationship between damage to the cerebellar peduncles and clinical disability in multiple sclerosis, Radiology 271 (2014), 822-830. doi:10.1148/radiol. 13132142.

[25] R.G. Quisling, A.J. Barkovich and B. Maria, Magnetic resonance imaging features and classification of central nervous system malformations in Joubert syndrome, J. Child Neurol. 14 (1999), 628-635. doi:10.1177/088307389901401002.

[26] K. Sasaki, H. Oka, Y. Matsuda, T. Shimono and N. Mizuno, Electrophysiological studies of the projections from the parietal association area to the cerebellar cortex, Exp. Brain Res. 23 (1975), 91-102. doi:10.1007/BF00238732.

[27] M.V. Spampinato, J. Kraas, B.L. Maria, Z.J. Walton and Z. Rumboldt, Absence of decussation of the superior cerebellar peduncles in patients with Joubert syndrome, Am. J. Med. Genet. A 146A (2008), 1389-1394.

[28] M. Thürling, H. Hautzel, M. Küper, M.R. Stefanescu, S. Maderwald, M.E. Ladd et al., Involvement of the cerebellar cortex and nuclei in verbal and visuospatial working memory: A 7T fMRI study, Neuroimage 62 (2012), 1537-1550. doi:10.1016/j.neuroimage.2012.05.037.

[29] K.E. Travis, Y. Leitner, H.M. Feldman and M. Ben-Shachar, Cerebellar white matter pathways are associated with reading skills in children and adolescents, Hum. Brain Mapp. 36 (2015), 1536-1553. doi:10.1002/hbm.22721.

[30] Y. Tsuboi, J. Slowinski, K.A. Josephs, W.G. Honer, Z.K. Wszolek and D.W. Dickson, Atrophy of superior cerebellar peduncle in progressive supranuclear palsy, Neurology 60 (2003), 1766-1769. doi:10.1212/01.WNL.0000068011.21396. F4.

[31] A. Uchino, A. Sawada, Y. Takase and S. Kudo, Symmetrical lesions of the middle cerebellar peduncle: MR imaging and differential diagnosis, Magn. Reson. Med. Sci. 15 (2004), 133-140. doi:10.2463/mrms.3.133.

[32] F. Wang, Z. Sun, X. Du, X. Wang, Z. Cong and H. Zhang et al., A diffusion tensor imaging study of middle and superior cerebellar peduncle in male patients with schizophrenia, Neurosci. Lett. 48 (2003), 35-38.

[33] F. Xu and D.T. Frazier, Role of the cerebellar deep nuclei in respiratory modulation, Cerebellum 1 (2002), 35-40. doi:10. 1080/147342202753203078.

[34] Z.H. Zheng, E. Dietrichs and F. Walberg, Cerebellar afferent fibres from the dorsal vagal nucleus in the cat, Neurosci. Lett. 32 (1982), 113-118. doi:10.1016/0304-3940(82)90259-2.

[35] J.N. Zhu, W.H. Yung, B. Kwok-Chong Chow, Y.S. Chan and J.J. Wang, The cerebellar-hypothalamic circuits: Potential pathways underlying cerebellar involvement in somatic-visceral integration, Brain Res. Rev. 52 (2006), 93-106. doi:10. 1016/j.brainresrev.2006.01.003.

[36] J. Zhuang, F. Xu and D.T. Frazier, Hyperventilation evoked by activation of the vicinity of the caudal inferior olivary nucleus depends on the fastigial nucleus in anesthetized rats, J. Appl. Physiol. 4 (2008), 1351-1358. doi:10.1152/ japplphysiol.00824.2007. 